\title{
Long term outcome of transanal endorectal pull-through for Hirschsprung's disease: A single institute experience
}

\author{
T Gobran, $M D$; M Khalifa, $M D$; $\boldsymbol{A}$ Waly, $M D$
}

Department of General Surgery, Pediatric Surgery Unit, Zagazig University, Egypt.

\begin{abstract}
Background: The transanal endorectal pull-through operation (TEPT) for Hirschsprung's disease (HD) is relatively new and this makes assessment of the functional outcome difficult. The aim of this study was to assess the long-term functional outcome of TEPT for short segment HD (at Faculty of Medicine-Zagazig University) and to evaluate its effect on the patients' quality of life.

Patients and methods: Fifty-eight patients out of 176 patients who underwent TEPT technique for HD between August 2002 to August 2009 were followed up for at least one year. All patients had aganglionic segment that is confined to the rectosigmoid area. Long term outcome and quality of life were assessed by interviews with the parents and/or patients using pre-structured questionnaires filled by the attending doctor.

Results: Forty-four patients had satisfactory results without complications. Reported postoperative complications included soiling in 11 patients, constipation in 3 patients, incontinence in 3 patients and enterocolitis in 7 patients. According to quality of life scoring criteria, 75.9\% of patients had good quality of life and $18.9 \%$ had fair quality of life.

Conclusion: TEPT for short segment HD is associated with gradual recovery of stooling pattern and long term normal bowel function and good quality of life for patients.

Key words: Hirschsprung's disease, long term follow up, quality of life, fecal soiling, constipation, fecal incontinence.
\end{abstract}

\section{Introduction:}

Hirschsprung's disease (HD) is a common neurogenic cause of functional neonatal intestinal obstruction affecting approximately 1:5000 births. ${ }^{1}$ Aganglionosis is confined to rectosigmoid segment in approximately $80 \%$, but proximal extension occurs and up to $15 \%$ of patients have total colonic aganglionosis. ${ }^{2}$ Surgical treatment of HD has changed in recent decades, with attempts made to reduce extensive surgical dissections to reduce postoperative complications and hospitalization time and ultimately decreasing morbidity. ${ }^{3}$

Endorectal pull-through was described in 1964 by Soave. ${ }^{4}$ In the same year, the technique was modified by Boley who performed the colo-anal anastomosis during the pull through. ${ }^{5}$ Transanal endorectal pull through (TEPT) represents the latest development in the concept of the minimally invasive surgery for HD. ${ }^{6}$
Since the description of TEPT for HD by De la Torre Mondragon and Ortega Salgado, ${ }^{7}$ the approach has become commonly used by pediatric surgeons. ${ }^{8}$ Compared with the traditional approaches such as the Swenson, Duhamel, and Soave, the most advantage of the operation is that it is minimally invasive and eliminates the abdominal incision resulting in no abdominal scar and complications of traditional laparotomy. The operating time and hospital delay are also cut short. Furthermore, the anal sphincter is kept in operation so that the morbidity of postoperative incontinence is sharply cut down. It has become increasingly popular to more and more doctors and patients. ${ }^{9}$

The long term follow-up of children with HD gives one the best opportunity to critically evaluate the efficacy and results of a particular surgical procedure. In general, the most commonly encountered problems include 
constipation, incontinence, enterocolitis and the overall impact of the disease on lifestyle of patients and/or their families. ${ }^{10}$ The aim of this study was to assess the long-term functional outcome of TEPT for short segment HD (at Faculty of Medicine-Zagazig University) and to evaluate its effect on the patients' quality of life.

\section{Patients and methods:}

A retrospective review of medical records revealed 176 children who had undergone transanal endorectal pull-through (TEPT) for correction of short segment HD in Pediatric Surgery Unit- Faculty of Medicine-Zagazig University, between August 2002 to August 2009. Of all these children 58 patients were included and 118 were lost.

\section{Exclusion criteria were:}

- Associated Down's syndrome, mental retardation, or cerebral palsy.

- Less than one year follow-up.

- Patients who refused to complete the questionnaire,

All information about clinical, operative and postoperative data were obtained from medical records including gender, age at time of surgery, mode of presentation, details of surgery, results of early postoperative follow up visits and postoperative complications encountered including anastomotic leakage, stricture and enterocolitis. The next step was to invite families to participate in the study by telephone. The patients and their parents were interviewed by a pre-structured questionnaire. Questions were asked to collect data on stooling pattern (stool frequency, stool consistency, stool control), fecal soiling, constipation, incontinence, medication use enterocolitis, and the effect of bowel habits on the child's activities and social life.

The clinical examination included a digital rectal examination that assessed the presence or absence of rectal prolapse, tone of the anal sphincter, ampulla capacity, amount of feces, and anastomosis.

Long-term outcome was evaluated by a clinical bowel function scoring system Table(1) ${ }^{11}$ and quality of life was assessed according to the Quality of Life Scoring Criteria for children with fecal incontinence Table(2). ${ }^{12}$

Table (1): Clinical bowel function scoring system.

\begin{tabular}{|l|l|c|}
\hline Item & Criteria & Points \\
\hline \multirow{2}{*}{ Frequency of defecation } & Every 2 d or 1-2/d & 2 \\
& $3-5 /$ d or 2 to 3/wk & 1 \\
& $>1 /$ wk or $>5 / \mathrm{d}$ & 0 \\
\hline \multirow{2}{*}{ Soiling } & Absent & 4 \\
& Accidental & 3 \\
& Frequent & 2 \\
\hline \multirow{2}{*}{ Incontinence } & Accidental & 1 \\
& Frequent & 0 \\
\hline \multirow{2}{*}{ Fecal sensation } & Normal & 2 \\
& Defective & 1 \\
& Missing & 0 \\
\hline \multirow{2}{*}{ Pain or difficulty with defecation } & Never & 2 \\
& Accidental & 1 \\
& Frequent & 0 \\
\hline Fecal consistency & Normal & 2 \\
& Loose & 1 \\
& Liquid & 0 \\
\hline
\end{tabular}

NOTE: Good, 9 to 12 points; fair, 5 to 8 points; poor, 0 to 4 points. 
Table (2): Quality of life scoring criteria for children.

\begin{tabular}{|l|l|c|}
\hline Item & Criteria & Points \\
\hline \multirow{2}{*}{ Soiling } & Absent & 4 \\
& Accidental & 3 \\
& Frequent & 2 \\
\hline Incontinence & Accidental & 1 \\
& Frequent & 0 \\
\hline School absence & Never & 2 \\
& Accidental & 1 \\
& Frequent & 0 \\
\hline Unhappy or anxious & Never & 2 \\
& Accidental & 1 \\
& Frequent & 0 \\
\hline Food restriction & No & 2 \\
& Somewhat & 1 \\
& Much & 0 \\
\hline Peer rejection & Never & 2 \\
& Accidental & 1 \\
& Frequent & 0 \\
\hline
\end{tabular}

NOTE: Good, 9 to 12 points; fair, 5 to 8 points; poor, 0 to 4 points.

\section{Results:}

This study included 58 patients who underwent TEPT technique during period from August 2002 to August 2009. The mean age at time of surgery was 3.5 years. The youngest age at operation time was one month age, whilst the oldest was 14 years. The data concerned with bowel function, stooling pattern and their effect on quality of life are shown in Table(3).

\section{Stool frequency}

Fifty patients had mean stool times 1 to 2 per day $(86.2 \%)$, only 5 patients $(8.6 \%)$ had mean stool times 3 to 5 per day and only 3 patients $(5.2 \%)$ had mean stool times 1 to 2 per week in the exact follow-up.

\section{Stool Control}

Forty one patients $(70.7 \%)$ had long-term normal bowel function and 17 patients $(29.3 \%)$ had long-term bowel dysfunction. Among these, $11 / 58$ patients $(18.9 \%)$ had fecal soiling, particularly at night. Three of total 58 patients (5.2\%) suffered from fecal incontinence and $3 / 58$ patients $(5.2 \%)$ had constipation.

\section{Stool Consistency}

Forty-nine patients $(84.4 \%)$ had a normal stool consistency. Six patients $(10.4 \%)$ had muddy foul smelling loose stools and frequent flatus. Three patients had liquid stools (5.2\%).

\section{Pain or difficulty with defecation}

Forty-five patients $(77.6 \%)$ had no pain with defecation. Ten patients $(17.2 \%)$ had accidental pain with defecation. Three patients $(5.2 \%)$ had frequent pain with defecation (including 3 patients who suffered from constipation).

\section{Late postoperative complications}

\subsection{Enterocolitis}

Seven cases $(12 \%)$ had postoperative enterocolitis (in the form of offensive diarrhea, fever, toxemia) during long term follow up period, two cases had mild attacks and five cases had moderate attacks and all these cases were hospitalized and responded to medical treatment in the form of intravenous fluids, parentral antibiotics, enemas, and metronidazole. 


\subsection{Anastomotic stricture}

There were $5(8.6 \%)$ cases of anastomotic stricture developed within weeks from surgery, 4 of them were infants at time of surgery. All these patients improved on regular anal dilatation during clinic visits and none of them required surgical intervention.

\subsection{Constipation}

It was found in 3 patients $(5.2 \%)$ in this study. Barium enema showed colonic dilation and revision of histopathology was done and detected residual aganglionic segment in two patients. The third patient had redundant colon. Redo transanal endorectal pull-through was feasible in two patients and the remainder one needed conversion transanal endorectal pullthrough. Of these three cases, two showed improvement of bowel function during the long-term follow up and one case showed true incontinence.

\subsection{Fecal soiling}

Postoperative soiling accidents were observed in 11 patients (18.9\%). These cases could be classified as having either heavy or light soiling based on frequency of episodes. Seven patients had light (accidental) soiling and four patients had heavy (frequent) soiling. These patients were treated with constipating diet and drugs and improved with time.

\subsection{Fecal incontinence}

Three patients $(5.2 \%)$ had fecal incontinence. They had a nondilated colon on contrast enema and frequent attacks of diarrhea and did not respond to medical treatment. Also these children were evaluated with revision histopathology and all of the results confirmed the presence of ganglion cells. The patients underwent anorectal manometry which revealed decreased basal resting pressure (BRP), maximal squeeze pressures (MSP) voluntary sphincter force (VSF) and negative rectoanal relaxation reflex.

\subsection{Mortality}

No mortality was death recorded during the follow up period.

\section{Quality of life}

Because of fecal soiling or incontinence, 14 patients $(24.1 \%)$ had to restrict their foods. School absence occurred in 5 patients $(8.6 \%)$ as patients limited their physical activity because of soiling or odor. Five patients $(8.6 \%)$ had problems in peer relationships. Three patients remained medical therapy dependent and $11(18.9 \%)$ patients needed occasional intermittent therapy, such as medication, enema, and diapers. Forty-four patients (75.9\%) had good quality of life and no limitation to their social activities. Eleven patients (18.9\%) had fair quality of life and $3(5.2 \%)$ had poor quality of life.

\section{Discussion:}

The goal of treating a child with HD should be to achieve anorectal function that is as near to normal as possible. The best approach is to bring ganglionic bowel down to a point just above the dentate line. ${ }^{13}$ The transanal endorectal pull-through operation is a relatively new minimally invasive technique. It leaves no abdominal incision or scar, avoids the potential complications of laparotomy (adhesions, wound infection), and is associated with shorter operating time and hospital stay. These advantages make it superior to traditional laparotomy in the immediate term. ${ }^{14}$ The functional outcome in patients treated for HD is variable. Many reports showed that the results of surgery for HD were satisfactory. ${ }^{11}$ The majority of long-term follow-up studies concentrated on the functional outcome and little is known about the quality of life of patients after surgical treatment for HD. The aim of this study was to assess the long-term functional outcome of TEPT for short segment HD (at Faculty of Medicine-Zagazig University) and to evaluate its effect on the patients' quality of life. 
Table (3): Bowel function, stooling pattern and quality of life for study cases.

\begin{tabular}{|l|c|c|}
\hline Items & Number & Percent \\
\hline Bowel function & & \\
Normal & 41 & 70.7 \\
Abnormal & 17 & 29.3 \\
Stool frequency & 50 & 86.2 \\
1-2 time/day & 5 & 8.6 \\
3-5 time/ day & 3 & 5.2 \\
1-2 time/ week & & 84.4 \\
Stool consistency & 49 & 10.4 \\
Normal & 6 & 5.2 \\
Liquid & 3 & 77.6 \\
Pain or difficulty of defecation & 45 & 17.2 \\
Never & 10 & 5.2 \\
Accidintal & 3 & 18.9 \\
Frequent & 11 & 6.9 \\
Fecal soiling & 4 & 12 \\
Heavy soiling & 7 & 5.2 \\
Light soiling & 3 & 12 \\
Fecal incontinence & 7 & 8.6 \\
Enterocolitis & 4 & \\
Anastomotic stricture & 3 & 5.2 \\
& 5.9 & 0 \\
Constipation & & 5.9 \\
Mortality & & \\
Quality of life & & \\
Good & & \\
Pair & & \\
\hline
\end{tabular}

Stooling patterns remains poorly understood in the most of the reported series after surgical correction of HD. A previous evaluation of stooling patterns in patients after endorectal pull-through (ERPT) clearly showed a return to normal stooling frequency over time. ${ }^{15}$ Although several authors of primary pullthrough series claim that their patients are continent this must be viewed with caution, because follow-up in many of these patients was no longer than 2 or 3 years. ${ }^{16}$ In this study, the patients were followed up from 1 to 7 years to evaluate the functional results of the operation as well as its impact on the quality of patients' life.

Postoperative bowel dysfunction had been reported to occur in $10 \%$ to $30 \%$ of patients with HD. ${ }^{11}$ Incontinence and constipation are 2 common reported problematic sources after surgery for HD. ${ }^{17}$ In our study, $(29.3 \%)$ of the patients had long-term bowel dysfunction in the form of soiling, incontinence and constipation. Soiling occurred in 11 patients $(18.9 \%)$, constipation in 3 patients $(5.2 \%)$ and incontinence in 3 patients $(5.2 \%)$. Zhang et $\mathrm{al}^{9}$ studied 58 patients who underwent transanal pull through operation for HD. The results were soiling in 9 patients $(15.5 \%)$ and constipation in $5(8.6 \%)$ while incontinence did not complicate any patient.

In this study, there were 5 cases $(8.6 \%)$ of anastomotic stricture with various degrees of 
constipation who improved on regular dilatation. These complaints did not develop until few weeks to two months after the pullthrough procedure and improved within one year after operation. One study reported overall incidence $14 \%$ anastomotic stricture of total 84 infants who were managed by TEPT for surgical correction of HD. Ten cases in that study improved by regular dilatation and 2 cases required surgical intervention. 18 So we recommend prophylactic anal bouginage with Hegar probe at 2 weeks after operation particularly in infants and neonates.

In the current study, persistent constipation was found in 3 patients $(5.2 \%)$, which is relatively low if compared to other studies such as Teitelbaum and colleagues work. ${ }^{13}$ They described their experience with 78 infants who were treated with TEPT, and reported constipation at a rate of $28 \%$. Van Leeuwen et al ${ }^{19}$ reported constipation in $22 \%$ of cases and El-Sawaf et al. ${ }^{20}$ found it in $(29.3 \%)$ of total 41 patients who underwent TEPT for HD.

In children with persistent constipation redo pull-through operation and resection of the problematic dilated bowel may be required. Indications for a second pull-through include retained or acquired aganglionosis, severe stricture, dysfunctional bowel segment, marked dilation of the bowel as a result of years of constipation, anocutaneous fistula, and intestinal neuronal dysplasia. ${ }^{21}$ In the present study, for the 3 patients with persistent constipation and who did not respond to conservative management in the form of laxatives and diet modification; barium enema and revision of histopathology were done and detected residual aganglionic segment in two patients, while the third patient had redundant dilated colon. Redo transanal endorectal pullthrough was performed to two patients with resection of the affected segment, while transabdomenal pull-through was performed to the third patient. Two cases showed improvement of bowel function during the long-term follow up and one case showed fecal incontinence. There are other studies that reported cases required redo pull-through after surgical correction of HD. Aggarwal et $\mathrm{al}^{22}$ performed redo pull-through procedures for four cases. The frequency of defecation in their four patients following a second pull-through operation was high in the early postoperative period. However, the stooling pattern improved considerably over the next few months. Teitelbaum and Coran, ${ }^{23}$ described an experience with 26 redo pull-through with a mean follow-up of 14 years. Almost all patients were continent except 2 that had daily leakage of stool. Although one patient required a third pull-through procedure, the authors concluded redo procedures can be performed effectively and yield good to excellent results.

Fecal incontinence after operative management of HD is a devastating complication. ${ }^{9}$ The exact cause of possible incontinence after TEPT operation is still unclear, it may be due to very low transanal dissection which may result in poor sphincter function and poor or absent sensation. ${ }^{24}$ In our study, 3 patients $(5.2 \%)$ had fecal incontinence and all of them had nondilated colon on contrast enema and frequent episodes of diarrhea and did not respond to medical treatment and dietary modifications. Zhang et $\mathrm{al}^{14}$ and Elhalaby et $\mathrm{al}^{8}$ did not report fecal incontinence post TEPT operation in their series. The occurrence of incontinence in our study may be due to the learning curve effect on the results (i.e., poorer results with procedures done earlier in the analysis).

Fecal soiling is one of the common problems occurring after surgical correction of HD which has significant impact on patient' quality of life and social development especially patients of school age. ${ }^{12}$ In our study fecal soiling occurred in (18.9\%) of patients postoperatively and $(6.9 \%)$ had heavy soiling. However, gradual decline could be noted in the rates of soiling seen in the immediate postoperative period in most patients, gradually improved with time. Elhalaby and his colleagues 8 reported transient soiling and increases in bowel movements in a significant number of their patients who underwent TEPT, and they felt the cause was the overstretching. However, they stated that this soiling was transient.

Enterocolitis has been considered one of the main problems in patients with HD both before and after definitive treatment. It was noticed that incidence of postoperative enterocolitis in this series was relatively low 
in comparison to other series. ${ }^{19,20,25}$ This may be due to our routine use of anal dilatation 2 weeks postoperatively particularly in infants and neonates, also underestimation in diagnosing early cases of enterocolitis because most of our patients' families are of low socioeconomic level and lack awareness of general condition of their children and reside far away from the hospital so usually were managed by general practitioners in primary health care clinics as a case of gastroenteritis.

There is a strong association between poor continence and negative effects on the child's social life and activities, most significant between 5 and 15 years of age. These are the formative years when a child begins to develop peer relationships and self-esteem. ${ }^{26}$ In our study $(75.9 \%)$ of patients had good quality of life and no limitation to their social activities. This is a reflection of the improved bowel function on long term follow-up of HD cases managed by TEPT. Eleven patients $(18.9 \%)$ had fair quality of life and $3(5.2 \%)$ had poor quality of life and were not satisfied. Bai et al ${ }^{11}$ studied forty-five patients who underwent the Swenson procedure for Hirschsprung's disease and reported eighteen patients (40\%) had good quality of life and, twenty-one patients $(46.7 \%)$ had fair quality of life, and 6 $(13.3 \%)$ had poor quality of life, according to Quality of- Life Scoring Criteria for children. These results indicate the importance of addressing and managing problems of fecal control over long-term follow-up visits of children with Hirschsprung's disease.

\section{Conclusion:}

TEPT for short segment HD is associated with gradual recovery of stooling pattern, long term normal bowel function and good quality of life for patients.

\section{References:}

1- Escobar M, Grosfeld J, West K, et al: Longterm outcomes in total colonic aganglionosis: A 32-year experience. $J$ Pediatr Surg 2005; 40: 955-961.

2- Cusick E, Woodward M: Hirschsprung's disease: Outcome and how to follow-up. Current Paediatrics 2001; 11: 286-290.

3- Takegawa B, Ortolan E, Rodrigues A, et al:
Experimental model for transanal endorectal pull-through surgery. Technique of De la Torre and Ortega . J Pediatr Surg 2005; 40: 1539-1541.

4- Soave F: A new surgical technique for the treatment of Hirschsprung's disease. Surgery 1964; 56: 1007-1044.

5- Boley SJ: New modification of the surgical treatment of Hirschsprung's disease. Surgery 1964; 56: 1015-1017.

6- Teeraratkul S: Transanal one-stage endorectal pull-through for Hirschsprung's disease in infants and children. $J$ Pediatr Surg 2003; 38: 184-187.

7- De la Torre-Mondragon L, Ortega-Salago JA: Transanal endorectal pull-through for Hirschsprung disease. J Pediatr Surg 1998; 33: 1283-1286.

8- Elhalaby E, Hashish A, Elbarbary MM, et al: Transanal one-stage endorectal pullthrough for Hirschsprung's disease: A multicenter study. J Pediatr Surg 2004; 39: 345-351.

9- Zhang S, Bai Y, Wang W, et al: Clinical outcome in children after transanal 1- stage endorectal pull-through operation for Hirschsprung disease. J Pediatr Surg 2005; 40: 1307-1311 .

10-Moore S, Albertyn R, Cywes S: Clinical outcome and long-term quality of life after surgical correction of Hirschsprung's disease. J Pediatr Surg 1996; 31: 14961502.

11-Bai Y, Chen H, Hao J, et al: Long-term outcome and quality of life after the Swenson procedure for Hirschsprung's disease. J Pediatr Surg 2002; 37: 639- 642.

12-Bai Y, Yuan Z, Wang W, et al: Quality of life for children with fecal incontinence after surgically corrected anorectal malformation. J Pediatr Surg 2000; 35: 462- 464.

13-Teitelbaum H, Cilley R, Sherman J, et al: A decade of experience with the primary pull-through for Hirschsprung disease in the newborn period: A multicenter analysis of outcomes. Ann Surg 2000; 232: 372380 .

14-Zhang S C, BaiY Z, Wang W, Wang W L: Stooling patterns and colonic motility after transanal one-stage pull-through 
operation for Hirschsprung's disease in children. J Pediatr Surg 2005; 40: 17661772.

15-Teitelbaum D, Drongowski R, Chamberlain $\mathrm{J}$, Coran A: Long-term stooling patterns in infants undergoing a primary endorectal pullthrough (ERPT) for Hirschsprung's disease. J Pediatr Surg 1997; 32: 10491053.

16-Cass D: Aganglionosis: associated anomalies. J Paediatr Child Health 1990; 26: 351-354.

17-Keshtgar S, Ward H, Clayden G, et al: Investigations for incontinence and constipation after surgery for Hirschsprung's disease in children. Pediatr Surg Int 2003; 19(1-2): 4-8.

18-Rouzrokh M, Khaleghnejad A T, Mohejerzadeh L, Heydari A Molaei H: What is the most common complication after one-stage transanal pull-through in infants with Hirschsprungís disease? Pediatr Surg Int 2010; 26: 967-970.

19-Van Leeuwen K, Geiger J, Barnett L, et al: Stooling and manometric findings after primary pull-through in Hirschsprung's disease: Perineal versus abdominal approaches. J Pediatr Surg 2002; 37(9): 1321-1325.
20-El-Sawaf M, Drongowski R, Chamberlain $\mathrm{J}$, et al: Are the long-term results of the transanal pull-through equal to those of the transabdominal pullthrough? J Pediatr Surg 2007; 09.007.

21-Engum, Grosfeld: Long-term results of treatment. Seminars in Pediatric Surgery 2004; 13(4).

22-Aggarwal S, Yadav S, Goel D, et al: Combined abdominal and posterior sagittal approach for redo pull-through operation in Hirschsprung's disease. J Pediatr Surg 2002; 37: 1156-1159.

23-Teitelbaum H, Coran A: Reoperative surgery for Hirschsprung's disease. Sem Pediatr Surg 2003; 12: 124-131.

24-Levitt M, Martin C, Olesevich M, et al: Hirschsprung disease and fecal incontinence: Diagnostic and management strategies. J Pediatr 2009; 127: 954-957.

25-Zhang S, Wang W, Bai Y, et al: Determination of total and segmental colonic transit time in children after surgery for Hirschsprung disease. Chin J Pediatr Surg 2003; 24(2): 119-121.

26-Needlman R: Growth and development. In: Textbook of pediatrics. Nelson WE, Bebrman RE, Kliegman RM, et al (Editors); Philadelphia, PA Saunders (Publisher); edn. 15; 1996; p. 30-72. 